\title{
Lithium Induced Birth Defect
}

National Cancer Institute

\section{Source}

National Cancer Institute. Lithium Induced Birth Defect. NCI Thesaurus. Code C98974.

Cong enital abnormalities, mainly cardiovascular malformations, which develop in a fetus when the mother uses lithium medication during pregnancy. 